\title{
Personal values in mental disorders: an exploratory analysis
}

\author{
Valentina Socci ${ }^{1 凶}$, Dalila Talevi ${ }^{1}$, Paolo Stratta ${ }^{2}$, Alessandro Rossi ${ }^{1,2}$, Francesca Pacitti (10 1,2, Alessia Lucaselli ${ }^{1}$, \\ Eleonora Gregori', Eleonora Quarta ${ }^{1} \&$ Rodolfo Rossi ${ }^{3}$
}

Personal values have increasingly become central in socio-psychological research. However, the relationships between values and psychopathological variables have been scarcely investigated, with mixed results. This study aimed to explore potential differences in value orientation in a sample of people with psychotic disorders and mood disorders compared to a non-clinical sample using the Schwarz's values framework. A clinical sample of 162 subjects (92 subjects with psychotic disorders and 70 with mood disorders) and a non-clinical sample of 217 subjects completed the self-report measures of affective states (PANAS) and personal values (PVQ). Irrespective of the diagnostic group, the clinical sample showed higher expression of Conservation values (i.e., Tradition, Conformity, Security). Conservation and Self-enhancement values positively correlated with PANAS-positive affectivity in the clinical sample; in the non-clinical sample, Self-enhancement values only correlated with PANASpositive affectivity. The expression of Conservation values in individuals with a mental disorder could reflect an orientation toward Conformity underlying fundamental affiliative goals. The complex relationship between personal values and clinical constructs should be further investigated, with important theoretical and clinical implications in mental health.

\footnotetext{
${ }^{1}$ Department of Biotechnological and Applied Clinical Sciences, University of L'Aquila, L'Aquila, Italy. ${ }^{2}$ Department of Mental Health, ASL1, L'Aquila, Italy.

${ }^{3}$ Department of Systems Medicine, University of Tor Vergata, Rome, Italy. ${ }^{凶}$ email: valentinasocci@gmail.com
} 


\section{Introduction}

$\mathrm{n}$ recent years, personal values have become central in many socio-psychological research domains, with a growing body of research investigating the development, structure and implications of human values in different cultures (Sagiv et al., 2017; Schwartz and Bardi, 2001).

According to Schwartz (1992), basic human values can be defined as broad, trans-situational and desirable goals that represent guiding principles in people's lives (Schwartz, 1992). Schwartz identified ten basic values, each one expressing a different motivational goal, grounded to three universal requirements of human existence, i.e., organic biological needs, requisites of coordinated social interactions and welfare needs of groups and society. This theory describes a structure of relations among values in a circular continuum, in which some of the values are mutually compatible, positively correlated and therefore adjacent in the circle. In contrast, opposite values are less positively or negatively correlated, thus expressing conflicting motivational goals (Schwartz, 2012) (Fig. 1). Schwartz's model can be summarized in its circular structure resulting in four higher-order values that express two fundamental conflicts. Specifically, "Selfenhancement" values, that refer to the self-interest in controlling people and resources (i.e., Power) or ambition and need for gaining socially recognized success (i.e., Achievement), contrast with "Self-transcendence" values, that express concern for the welfare of individuals (i.e., Benevolence) and tolerance and social acceptance (i.e., Universalism). Similarly, "Openness to change", concerning the personal motivation for autonomy (i.e., Selfdirection) and novelty seeking (i.e., Stimulation), conflicts with "Conservation" values, that relate to the motivation to preserve the status quo maintaining traditional customs and beliefs (i.e., Tradition), to comply with rules and other's expectations (i.e., Conformity) and to seek safety and stability (i.e., Security) (Schwartz, 1992, 2012).

This set of basic values has been used to explain a variety of attitudes and behaviors, mainly due to the heuristic and predictive power of Schwartz's circular model (Schwartz and Bardi, 2001; Schwartz et al., 2012). Indeed, personal values have been conceptualized as mental representations of desirable goals that

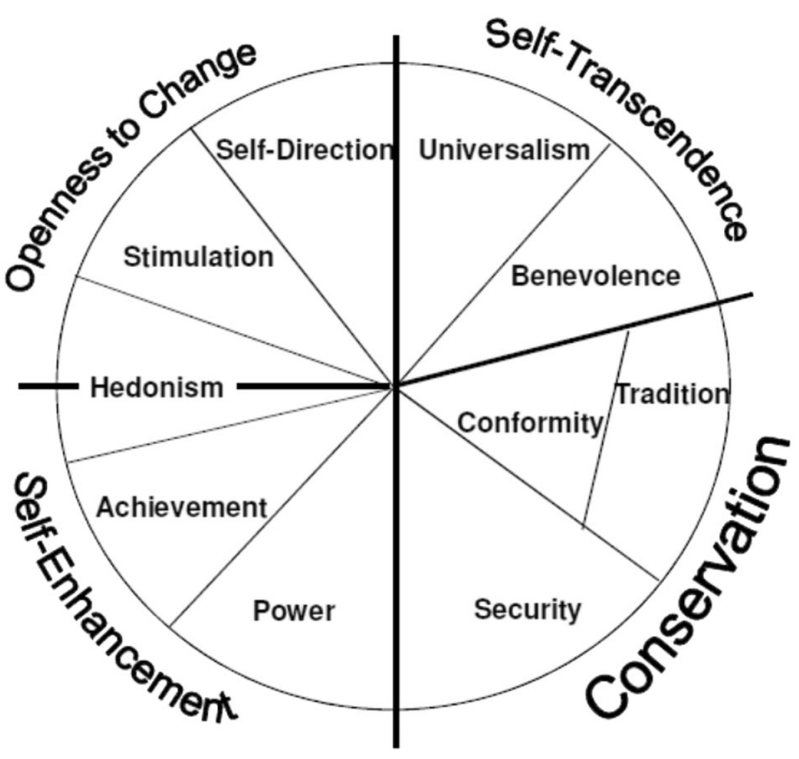

Fig. 1 The value model of Schwartz (2012). The circular structure describes relations of conflict and congruity among ten basic values resulting in four higher-order values. promote, through motivational and cognitive processes, corresponding value-expressive behaviors (Bardi and Schwartz, 2003).

As personal values are central in the construction of self-image, they are believed to modulate an individual's thoughts, attitudes, decisions and behaviors. Accordingly, several lines of research investigated values orientation, attitudes and behaviors (Sagiv et al., 2017) in the context of positive affectivity, subjective wellbeing (e.g., Sagiv and Schwartz, 2000; Roccas et al., 2002) and prosocial behavior toward close or distant others (Sagiv et al., 2017 for a review). In this respect, Self-transcendence values such as Benevolence and Universalism were related to concern for others (Schwartz, 1992; Bardi and Schwartz, 2003) and tolerance toward various minority groups (Davidov and Meuleman, 2012; Kuntz et al., 2015). Conversely, an association between Power and Stimulation values with positive attitudes toward interpersonal violence has been suggested (Seddig and Davidov, 2018).

\section{Relations between personal values and psychopathology}

Personal values have been classically conceptualized as closely linked to subjective well-being, depending on the specific motivational orientation they express (Bilsky and Schwartz, 1994; Schwartz and Sortheix, 2018). Within this framework, values related to growth and self-expansion (i.e., Universalism, Benevolence, Self-direction, Stimulation, Achievement) are thought to be more strongly associated with positive affectivity and wellbeing. In contrast, self-protective values related to deficiency needs (i.e., Tradition, Conformity and Security) may be associated with negative affectivity (see Schwartz and Sortheix, 2018, for a recent review).

Only a minority of investigations have assessed the association between values and negative affectivity or psychopathological variables. Jarden (2010) showed negative relations of Self-direction, Stimulation and Hedonism values with depressed mood (Jarden, 2010); conversely, no associations between power/ materialism nor security/hedonism and depression have been revealed in a sample of native American adolescents, while negative relations have been shown between depression and tradition/benevolence values (Mousseau, Scott and Estes, 2014). Hedonism and Stimulation predicted lower Post-traumatic Stress Disorder (PTSD) symptoms among crime victims (Maercker et al., 2009). Similarly, Power and Hedonism were negatively correlated with the probability and severity of PTSD in a sample of German soldiers (Zimmermann et al., 2014). On the other hand, traditional values such as Tradition and Universalism were detrimental to these PTSD populations (Maercker et al., 2009; Zimmermann et al., 2014). Results from a study by Hanel and Wolfradt (2016) showed that conservative/collectivistic values such as Tradition and Conformity are mostly unrelated to a set of subdimensions of different clinical variables (i.e., anxiety, depression, stress and schizotypy) (Hanel and Wolfradt, 2016). Moreover, a multinational student study (Maercker et al., 2015) showed that personal values predicted mental health indicators, with traditional values predicting mental health mediated by social support and modern values predicting mental health mediated by resilience. Collectively, these investigations suggest a link between values and clinical variables in the general population.

Recently, Schwartz's human values frame has been applied to a clinical sample of people with mental disorders (Rossi et al., 2020). Specifically, the study aimed at investigating the relationship between human values and variables related to interpersonal violence in subjects with psychotic disorders, mood disorders and personality disorders. Results showed that Self-enhancement and Openness to change values such as Stimulation and Achievement 
were positively related to self-reported interpersonal violence, while Conservation values were positively related to self-reported parental psychological support; moreover, this pattern of relations was similar in individuals with psychosis and personality disorders, while the correlations were weaker in the mood disorder sample. This study therefore suggests that personal values can be positively related to negative behaviors such as interpersonal violence in a mental illness sample.

The causal link between extremely deviant values and psychopathology is unclear. In this respect, evidence suggests that deviation of the motivational structure from its harmonious development could be associated with a specific pattern of relations among values across different clinical populations. Furthermore, since personal values can be conceptualized as emerging from a genetic heritage in conjunction with the exposure to social environments (Schwartz, 1992; Sagiv et al., 2017), alterations of value processing at the individual level could also affect the social affiliative system, thus increasing the susceptibility to mental disorders. Besides, cultural and contextual variables could modulate value orientation, potentially also influencing the relations between personal values and health. However, these arguments remain speculative, since current evidence concerning the relationship between personal values and mental health cannot be considered as conclusive. Evidence accumulated so far suggests the need to investigate further the value system and its contextual and cultural influences in mental illness samples.

On the basis of the above-mentioned results, we aimed to further explore the preexisting empirical support to the relations between values and psychopathology by investigating personal values in subjects with mental disorders compared to a nonclinical sample.

As personal values are highly desirable and attainable constructs variously related to positive affectivity of subjective wellbeing, we also used a measure of positive and negative affectivity to delineate the meaning of personal values further.

\section{Methods}

Study design. The study had a cross-sectional observational design. The local Institutional Review Board at the University of L'Aquila approved the investigation (approval number 38204). Written informed consent was obtained from each participant prior to inclusion. This study adheres to the Helsinki declaration.

Participants and data collection. Our sample included a total of 379 subjects from both clinical and non-clinical populations. The clinical sample included 164 consecutive adult subjects admitted to the Psychiatric Unit of "San Salvatore" Hospital during a 15 months' period, for an acute episode of psychotic or mood disorder. Primary diagnoses were determined at intake via clinician-administered interviews according to the Italian version of Diagnostic and Statistical Manual of Mental Disorders, fifth edition (DSM-5) (American Psychiatric Association, 2013). Patients who were unable to provide informed consent or to complete the study tasks due to the presence of language barriers, impaired consciousness, severe aphasia, intellectual disability or other cognitive deficits were excluded from participation in the study. Among eligible patients (n. 250), 86 refused to participate. Therefore, our final sample was of 164 patients. Ninety-two subjects were diagnosed as affected by psychotic disorders, 72 mood disorders. Subjects completed the self-report questionnaires at the scheduling of discharge or discharge, in order to minimize bias due to variations of clinical conditions. Data on age, gender, marital status, education level and occupational status were also collected.
The non-clinical sample included 217 undergraduate University students who accepted to fill in an online survey in Italian. The online recruitment took place for two months. We included people aged from 18 to 65 years. Then, a snowball sampling technique through the internet was used by asking participants to re-share the survey link on their social network page or via email to their acquaintances or family members. All answers were required in order to complete the survey.

\section{Measures}

The Portrait Values Questionnaire-40 (PVQ). Personal values were assessed using the PVQ in its Italian version (Capanna et al., 2005). Each of the 40 items consists of a short verbal portrait of 40 people, gender-matched with the respondent (Schwartz et al., 2001; Schwartz and Rubel, 2005). Each item describes the person's goals, aspirations and wishes, pointing therefore at one of the basic values as defined in the Schwarz's model. For each item, participants are asked to rate how similar to the portrait subject they are in a 6-point numerical scale ranging from "very similar" to "very dissimilar". Participant's values are, therefore, inferred by their self-report similarities judgments. The task assessed the ten basic values (Self-Direction; Stimulation; Hedonism; Achievement; Power; Security; Conformity; Tradition; Benevolence; Universalism) that were subsequently grouped into four higherorder values: Self-transcendence and Conservation, Openness to change and Self-enhancement values.

Positive and Negative Affect Schedule (PANAS). The Positive and Negative Affect Schedule (PANAS; Italian version; Terracciano, McCrae and Costa, 2003) is a self-report measure that assesses various types of positive and negative affective states. It is widely used to measure mood and emotions due to its excellent psychometric properties. The questionnaire includes two 10-item mood scales (PA: Positive Affects and NA: Negative Affects) consisting of words (e.g., interested, excited, distressed, upset). Participants are asked to rate how each of the words describes them right now. Ratings are provided on a 5-item Likert-type scale, from "very slightly or not at all" to "extremely".

Statistical analyses. Mean \pm SD and frequencies were calculated for descriptive analyses. After inspection for normality in row data, PVQ values were squared-transformed in order to account for non-normality. A one-way analysis of variance (ANOVA) with Bonferroni post hoc test was performed in order to assess differences between PVQ and PANAS (Table 1).

Pearson correlations between PANAS and PVQ values were performed separately for clinical and non-clinical samples. A Fisher's $r$ to $z$-transformation was performed in order to assess differences between correlation coefficients of the non-clinical vs. clinical sample. Data were analyzed using the Statistical Package for Social Sciences (SPSS, version 24, IBM, U.S.A.).

\section{Results}

One-hundred sixty-four patients accepted to be included in the study, but two subjects showed more than $5 \%$ of missing data and were therefore not involved in statistical computations; conversely, subjects of non-clinical sample showed no missing data. The final sample, therefore, included 217 undergraduate University students (non-clinical sample) and 162 patients (clinical sample).

The main socio-demographic characteristics of both clinical and non-clinical sample are summarized in Table 1. Most of the participants had an average education level (53.6\%), were single (54.8\%), whereas the $47.50 \%$ of the participants were employed/students. The $42.7 \%$ of the participants $(N=379)$ belonged to the clinical population $(n=162)$, whereas the $57.3 \%$ to the non-clinical population 
Table 1 Demographic characteristics of the clinical and non-clinical samples.

\begin{tabular}{|c|c|c|c|c|}
\hline Variables & Non-clinical $(n=217)$ & Psychotic disorders $(n=92)$ & Mood disorders $(n=70)$ & F-statistics $/ \chi^{2}$ \\
\hline Age & $36.38(10.38)$ & $39.51(12.34)$ & $48.34(10.79)$ & $F=31.51^{\star}$ \\
\hline \multicolumn{5}{|l|}{ Marital status } \\
\hline Single & 119 (54.8\%) & $68(73.9 \%)$ & $21(30 \%)$ & \multirow[t]{2}{*}{$\chi^{2}=65.36^{\star \star}$} \\
\hline Separated/divorced or widowed & $7(3.2 \%)$ & $9(9.8 \%)$ & $21(30 \%)$ & \\
\hline Education (years) & $16.16(3.41)$ & $12.05(3.92)$ & $11.99(3.99)$ & $F=59.40^{\star}$ \\
\hline \multicolumn{5}{|l|}{ Occupational status } \\
\hline Others (part-time work, stage) & $60(27.6 \%)$ & $17(18.5 \%)$ & $20(28.6 \%)$ & $\chi^{2}=96.91^{\star \star}$ \\
\hline
\end{tabular}

Table 2 Mean scores comparison (one-Way ANOVA with Bonferroni post hoc test) of values and affects in the non-clinical and clinical samples (mean \pm SD).

\begin{tabular}{lcccc} 
Variables & Non-clinical $(\boldsymbol{n}=\mathbf{2 1 7})$ & Psychotic disorders $(\boldsymbol{n}=\mathbf{9 2})$ & Mood disorders $(\boldsymbol{n}=\mathbf{7 0})$ & $\boldsymbol{F}-\mathbf{s t a t i s t i c s}$ \\
\hline PVQ & & & & \\
Self-transcendence & $4.77(0.63)$ & $4.77(0.80)$ & $4.90(0.71)$ & $\mathrm{F}(2,376)=0.94$ \\
Conservation $\mathrm{a}^{\star \star \star}, \mathrm{b}^{\star \star \star}$ & $3.99(0.64)$ & $4.40(0.90)$ & $4.47(0.80)$ & $\mathrm{F}(2,376)=16.30$ \\
Openness to change & $4.27(0.66)$ & $4.16(0.97)$ & $4.02(1.01)$ & $\mathrm{F}(2,376)=2.56$ \\
Self-enhancement & $3.56(0.95)$ & $3.27(0.99)$ & $3.14(1.00)$ & $\mathrm{F}(2,376)=6.1$ \\
PANAS & & & $29.83(10.23)$ & \\
Positive affect $\mathrm{t}^{\star \star}$ & $33.79(6.64)$ & $31.57(8.19)$ & $27.35(8.34)$ & $\mathrm{F}(2,376)=7.72$ \\
Negative affect $\mathrm{a}^{\star \star \star}, \mathrm{b}^{\star \star \star}, \mathrm{c}^{\star \star \star}$, & $19.41(7.39)$ & $22.53(9.38)$ & $\mathrm{F}(2,376)=26.25$
\end{tabular}

Descriptive values are reported as non-transformed values. ANOVA results were computed on squared-transformed values.

PVQ Portrait Values Questionnaire, PANAS Positive and Negative Affect Schedule.

${ }^{\star} p<0.05 ;{ }^{* \star} p<0.005 ;{ }^{\star \star \star} p<0.001$.

Post hoc Bonferroni comparisons: a Non-clinical vs. psychotic disorders.

bNon-clinical vs. mood disorders.

cPsychotic vs. mood disorders.

Table 3 Pearson correlations coefficient between PANAS and PVQ values in the non-clinical and clinical sample.

\begin{tabular}{|c|c|c|c|c|}
\hline Variables & PANAS positive & PANAS negative & PANAS positive & PANAS negative \\
\hline Openness to change & $0.324^{\star \star \star}$ & -0.008 & $0.571^{\star \star \star}$ & -0.118 \\
\hline Conservation & 0.094 & -0.081 & $0.277^{\star \star \star}$ & 0.052 \\
\hline Self-enhancement & $0.213^{\star \star}$ & $0.144^{\star}$ & $0.411^{\star \star \star}$ & 0.029 \\
\hline
\end{tabular}

$(n=217)$. Within the clinical sample, the $56.8 \%(n=92)$ was affected by psychoses and $43.2 \%(n=70)$ by mood disorders.

One-way ANOVA results showed a significant difference in Conservation $(\mathrm{F}(2,376)=16.30, p<0.001)$ between Non-Clinical vs. Psychotic disorders $(p<0.001)$ and Non-Clinical vs. Mood Disorders $(p<0.001)$ only, with no other differences observed in the remaining PVQ values (Table 2 ). This result did not change after covarying for age and education years.

\section{Correlations}

Since patients in the psychotic and mood disorders groups did not show differences in PVQ values mean scores, we estimated correlation coefficients in the clinical sample as a whole (Table 3). Significant correlations were found in both samples between values and Positive Affects but not with Negative Affects. In the non-clinical sample, Self-enhancement positively correlated with
Positive Affects $(r=0.21 ; p=0.002)$, whereas no correlations for Conservation were found. In the clinical sample, Selfenhancement positively correlated with Positive Affects $(r=0.41 ; p=0.000)$, as well as Conservation $(r=0.28$; $p=0.000)$. The correlation coefficients of Self-enhancement with Positive Affects showed a statistically significant difference between the clinical and non-clinical samples: $Z$-score $=-2.12$, $p=0.03$.

\section{Discussion}

In this study, we applied the Schwartz's basic values framework aiming to explore potential differences in value orientation among subjects with psychotic disorders and mood disorders compared to a non-clinical sample. Results showed that irrespective of the specific diagnosis, subjects in the clinical sample were characterized by higher Conservation value in comparison 
to the non-clinical sample. Moreover, results showed positive correlations between Conservation and Self-enhancement and PANAS positive affectivity in the clinical sample. In the nonclinical sample Self-enhancement only was related to positive affectivity.

Studies investigating personal values and clinical constructs suggested a link between values and mental health, with mixed results and some evidence supporting the existence of specific patterns of value orientation across different clinical groups (Maercker et al., 2009; Jarden, 2010; Mousseau et al., 2014; Zimmermann et al., 2014; Kjonius, Persson and Jonason, 2015; Hanel and Wolfradt, 2016; Rossi et al., 2018). In the present study, no specific pattern of values characterizing subjects with psychotic disorders and mood disorders were found; it seems therefore that the differences in value orientation are not specific to diagnostic domain; rather, these can be more spread out in a transdiagnostic way.

In the Schwartz's model (Schwartz, 1992), the motivational goals underlying the higher-order value Conservation entail a subordination of the self in favor of socially imposed expectations, resulting in a compliant social behavior (Bilsky and Schwartz, 1994). The attainment of these value types is mainly focused on avoiding conflicts contextually receiving approval, protection and support. Thus, Conservation values emphasize the pursuit of order and harmony in social relations that are crucial to maintaining social approval. Within this framework, the observed orientation toward Conservation values in the clinical sample could be viewed as the expression of conformity tendencies, which underlie fundamental affiliation-oriented goals.

Interestingly, the expression of Conservation values is associated with anxiety due to uncertainty in the social and physical world (Schwartz, 2012). An orientation toward values such as Conformity and Security is therefore expressed by people who experience feelings of unsafety, lack of control over their lives, and threat in relationships with others (Sagiv and Schwartz, 2000). Crucially, a close link between Conformity, social anxiety and self-esteem has been reported (Cialdini and Trost, 1998). As suggested, individuals frequently conform to others' expectations and beliefs to potentiate, protect or restore their self-esteem (see Cialdini and Goldstein, 2004) for a review). This could be additionally relevant for subjects with a severe mental illness, considering that low self-esteem and self-efficacy are inherently linked to mental disorders. Along with this reasoning, the orientation toward Conservation values expressed by the clinical sample could be interpreted in the context of social anxiety and/ or a loss of self-esteem related to the illness itself.

In this respect, although the exact magnitude of social influences on the individual expression of value remains partly underexplored, it has been shown that goals related to affiliation and social approval are strongly activated when one's self-esteem is threatened by the prospect or actual occurrence of not fitting in with the group (Cialdini and Goldstein, 2004). This could be particularly the case for the minority of individuals with mental disorders, an out-group frequently subjected to public stigma. Among the detrimental consequences of public stigma, people with mental disorders may endorse the negative stereotypes about mental illness (Corrigan and Watson, 2002). Internalized stigma, also referred to as self-stigma, typically leads to negative emotional reactions, mainly a loss of self-esteem and self-efficacy (Corrigan and Watson, 2002; Link et al., 2002; Lysaker, Roe and Yanos, 2007; Werner, Aviv and Barak, 2008; Marcussen et al., 2019), with a significant impact on individuals' goals and quality of life (Mashiach-Eizenberg et al., 2013; Oliveira et al., 2016). Interacting with specific personal resources and negative symptoms, internalized stigma was also associated with depression severity in schizophrenia (Rossi et al., 2017). Accordingly, stigmatization can be considered as an environmental risk factor for mental illness (Van Zelst, 2009).

Value orientation can be conceptualized as a flexible system reflecting changes in culture, society, and individual experience (Lane 1976). At the individual level, since personal values reflect basic psychological needs, their subjective importance could change over time depending on the actual or perceived fulfillment of these underlying needs. Therefore, it is conceivable that people with mental disorders, potentially experiencing social exclusion, could be particularly driven to conform in order to reinforce their self-esteem and sense of belongingness. Accordingly, a recent study on a non-clinical sample showed that traditional values (i.e., Security, Conformity, Tradition, Benevolence, Universalism) were associated with positive mental health, with social support being a mediator. As opposite, modern values (i.e., Self-direction, Stimulation, Hedonism, Achievement, Power) predicted positive mental health with the mediation of resilience. Moreover, higher Self-enhancement was found to be associated with a greater social distance (Maercker et al., 2015). Collectively, these findings point to Conservation as a potential way to defensively activate affiliative-oriented goals and corresponding behaviors, lowering the risk of social rejection, thus preventing social distance.

However, studies investigating human values have also shown possible detrimental effects of Conservation values on mental health (Bobowik et al., 2011; Oreg et al., 2008; Sagiv and Schwartz, 2000; Sverdlik and Oreg, 2015). Values such as Conformity and Security are believed to represent deficiency needs (Bilsky and Schwartz, 1994) and to correlate negatively with subjective well-being (Sagiv and Schwartz, 2000).

In this respect, our results showed that Self-enhancement values were correlated to PANAS positive affectivity in both clinical and non-clinical samples, thus confirming the association between values and subjective well-being. On the other hand, the lack of correlations between Conservation values and positive affectivity in the non-clinical sample partly supports the "unhealthy" nature of some values due to their intrinsic motivational content (see Schwartz and Sortheix, 2018, for a review).

However, the higher Conservation together with the correlation between Conservation and positive affect in the clinical sample is a new finding that partially supports higher "unhealthy" values in mental disorders but, on the other hand, suggest some adaptive meaning searching for self-protection and social approval.

Values with a strong self-protective, anxiety-based orientation such as Conservation could undermine subjective well-being by motivating a subordination of the self to socially imposed expectations, thus limiting autonomy (Schwartz and Sortheix, 2018). Therefore, the higher Conservation could represent the adaptive price paid by clinical subjects. As a matter of fact, Conformity could reflect avoidance or fear, which in turn represent risk factors for poor mental health (Ostermann et al., 2017).

In the present study, the observed relations between value orientation, clinical and non-clinical populations is still speculative, due to fundamental limitations inherent in the study design. Further, as a limitation, we did not include social inclusion and stigma evaluations, so that the suggested existence of a link between Conservation values, social inclusion and stigma in our clinical sample cannot be directly verified. Moreover, since in this study we included a clinical sample of subjects with mood disorders and psychotic disorders, the generalizability of the present results to other mental disorders is limited. These findings, therefore, should be further explored in a larger study including different diagnostic samples together with different "recovery" measures (Rossi et al., 2018). Lastly, the comparison with a sample of University students with no screening for common mental disorders represents a potential limitation of this study. Generalizing from student samples to the general public 
could also be problematic, particularly in socio-psychological research, when personality and attitudinal variables are considered (Hanel and Vione, 2016).

Nevertheless, the current study enriches the scarce literature on the relations between values and mental health, suggesting the potential existence of a common trajectory in the value orientation shared by people with mood disorders and psychotic disorders, i.e. the expression of Conservation values underlying fundamental affiliative-oriented goals. Further investigations are needed to confirm these findings. In this respect, the relations among personal values, personality traits, and different clinical constructs and recovery style (Huguelet et al., 2016; Kasai and Fukuda, 2017) should be further investigated, along with the impact of potential protective and risk factors. These could be relevant issues to be clarified, also for the potential implications in either theoretical and clinical domain (Bolton, 2010; Stanghellini and Ballerini, 2007). These results also underline the importance of including personal value assessment together with social inclusion evaluations in the clinical practice and rehabilitation process in the perspective of recovery-oriented care of individuals with mental illness (Huguelet et al., 2016; Talevi and Rossi, 2020; Treichler et al., 2019). Because of the exploratory nature of this study, further investigations are needed to better describe possible meaning of the personal values in mental health issues and outcomes.

\section{Data availability}

The datasets generated during and/or analyzed during the current study are not publicly available due to privacy reasons, but are available from the corresponding author on reasonable request.

Received: 25 February 2020; Accepted: 6 April 2021;

Published online: 04 May 2021

\section{References}

American Psychiatric Association (2013) Diagnostic and statistical manual of mental disorders, 5th edn. American Psychiatric Association

Bardi A, Schwartz SH (2003) Values and behavior: strength and structure of relations. Personal Soc Psychol Bull. https://doi.org/10.1177/0146167203254602

Bilsky W, Schwartz SH (1994) Values and personality. Eur J Pers 8(3):163-181. https://doi.org/10.1002/per.2410080303

Bobowik M, Basabe N, Páez D, Jiménez A, Bilbao ÁÁ (2011) Personal values and well-being among europeans, spanish natives and immigrants to Spain: does the culture matter? J Happiness Stud 12(3):401-419. https://doi.org/10.1007/ s10902-010-9202-1

Bolton D (2010) Conceptualisation of mental disorder and its personal meanings. J Ment Heal 19:328-336. https://doi.org/10.3109/09638237.2010.492483

Capanna C, Vecchione M, Schwartz SH (2005) La misura dei valori: Un contributo alla validazione del Portrait Values Questionnaire su un campione italiano [The measurement of values: A contribution to the validation of the Portrait Values Questionnaire on an Italian sample]. Bollettino di Psicologia Applicata $246: 29-41$

Cialdini RB, Trost MR (1998) Social influence: social norms, conformity, and compliance. In: Gilbert DT, Fiske ST, Lindzey G (eds) The Handbook of Social Psychology, 4th ed. Boston: McGraw-Hill, p 151-192

Cialdini RB, Goldstein NJ (2004) Social influence: compliance and conformity. Ann Rev Psychol 55(1):591-621. https://doi.org/10.1146/annurev. psych.55.090902.142015

Corrigan PW, Watson AC (2002) Understanding the impact of stigma on people with mental illness. World Psychiatry 1(1):16-20. Retrieved from http://www. ncbi.nlm.nih.gov/pubmed/16946807\%0A http://www.pubmedcentral.nih. gov/articlerender.fcgi?artid $=$ PMC1489832

Davidov E, Meuleman B (2012) Explaining attitudes towards immigration policies in European countries: The role of human values. J Ethn Migr Stud. https:// doi.org/10.1080/1369183X.2012.667985

Hanel PHP, Vione KC (2016) Do student samples provide an accurate estimate of the general public? PLoS ONE, 11(12). https://doi.org/10.1371/journal. pone. 0168354
Hanel PHP, Wolfradt U (2016) The "dark side" of personal values: Relations to clinical constructs and their implications. Pers Individ Dif 97:140-145. https://doi.org/10.1016/j.paid.2016.03.045

Huguelet P, Guillaume S, Vidal S, Mohr S, Courtet P, Villain L, Girod C, Hasler R, Prada P, Oliè E, Perroud N (2016) Values as determinant of meaning among patients with psychiatric disorders in the perspective of recovery. Sci Rep. https://doi.org/10.1038/srep27617

Jarden AJ (2010) Relationships between personal values, and depressed mood and subjective wellbeing. (Dissertation) University of Canterbury (Retrieved from http://ir.canterbury.ac.nz/handle/10092/5205).

Kasai K, Fukuda M (2017) Science of recovery in schizophrenia research: brain and psychological substrates of personalized value. NPJ Schizophr 3(1) https:// doi.org/10.1038/s41537-017-0016-6

Kuntz A, Davidov E, Schwartz SH, Schmidt P (2015) Human values, legal regulation, and approval of homosexuality in Europe: a cross-country comparison. Eur J Soc Psychol. https://doi.org/10.1002/ejsp.2068

Lane RE (1976) The Nature of Human Values. By Milton Rokeach. (New York: The Free Press, 1973. p. 438. \$13.95.). American Political Science Review 70 (3):965-966

Link BG, Struening EL, Neese-Todd S, Asmussen S, Phela JC (2002) Stigma as a barrier to recovery: the consequences of stigma for the self-esteem of people with mental illnesses. Psychiatr Serv 52(12):1621-1626. https://doi.org/ 10.1176/appi.ps.52.12.1621

Lysaker PH, Roe D, Yanos PT (2007) Toward understanding the insight paradox: Internalized stigma moderates the association between insight and social functioning, hope, and self-esteem among people with schizophrenia spectrum disorders. Schizophr Bull 33(1):192-199. https://doi.org/10.1093/ schbul/sbl016

Maercker A, Chi Zhang X, Gao Z, Kochetkov Y, Lu S, Sang Z, Yang S, Schneider S, Margraf J (2015) Personal value orientations as mediated predictors of mental health: A three-culture study of Chinese, Russian, and German university students. Int J Clin Heal Psychol 15(1):8-17. https://doi.org/10.1016/j. ijchp.2014.06.001

Maercker A, Mohiyeddini C, Müller M, Xie W, Yang ZH, Wang J, Müller J (2009) Traditional versus modern values, self-perceived interpersonal factors, and posttraumatic stress in Chinese and German crime victims. Psychol Psychother Theory Res Prac 82(2):219-232. https://doi.org/10.1348/147608308X380769

Marcussen K, Gallagher M, Ritter C, (2019) Mental Illness as a Stigmatized Identity. Society and Mental Health 9(2):211-227

Mashiach-Eizenberg M, Hasson-Ohayon I, Yanos PT, Lysaker PH, Roe D (2013) Internalized stigma and quality of life among persons with severe mental illness: the mediating roles of self-esteem and hope. Psychiatry Res 208 (1):15-20. https://doi.org/10.1016/j.psychres.2013.03.013

Mousseau AC, Scott WD, Estes D (2014) Values and depressive symptoms in American Indian youth of the northern plains: examining the potential moderating roles of outcome expectancies and perceived community values. J Youth Adolesc 43(3):426-436. https://doi.org/10.1007/s10964-013-9982-9

Oliveira SEH, Carvalho H, Esteves F (2016) Internalized stigma and quality of life domains among people with mental illness: the mediating role of self-esteem. J Ment Heal 25(1):55-61. https://doi.org/10.3109/09638237.2015.1124387

Oreg S, Bayazit M, Vakola M, Arciniega L, Armenakis A, Barkauskiene R et al. (2008) Dispositional resistance to change: measurement equivalence and the link to personal values across 17 nations. J Appl Psychol 93(4):935-944. https://doi.org/10.1037/0021-9010.93.4.935

Ostermann M, Huffziger S, Kleindienst N, Mata J, Schmahl C, Beierlein C, Bohus M, Lyssenko L (2017) Realization of personal values predicts mental health and satisfaction with life in a German population. J Soc Clin Psychol 36 (8):651-674. https://doi.org/10.1521/jscp.2017.36.8.651

Petri JK, Björn NP, Peter KJ, (2015) Hedonism, Achievement, and Power: Universal values that characterize the Dark Triad. Pers Individ Dif 77:173-178

Roccas S, Sagiv L, Schwartz SH, Knafo A (2002) The Big Five personality factors and personal values. Personal Soc Psychol Bull 28(6):789-801. https://doi. org $/ 10.1177 / 0146167202289008$

Rossi A et al. (2017) Personal resources and depression in schizophrenia: The role of self-esteem, resilience and internalized stigma. Psychiatry Research 256:359-364

Rossi A, Amore M, Galderisi S, Rocca P, Bertolino A, Aguglia E et al. (2018) The complex relationship between self-reported 'personal recovery' and clinical recovery in schizophrenia. Schizophr Res 192:108-112. https://doi.org/ 10.1016/j.schres.2017.04.040

Rossi A, Talevi D, Collazzoni A, Parnanzone S, Stratta P, Rossi R (2020) From basic human values to interpersonal violence: a mental illness sample. J Aggress Maltreatment Trauma 29(3):259-271. https://doi.org/10.1080/10926771.2019.1581865

Sagiv L, Roccas S, Cieciuch J, Schwartz SH (2017) Personal values in human life. Nat Hum Behav. https://doi.org/10.1038/s41562-017-0185-3

Sagiv L, Schwartz SH(2000) Value priorities and subjective well-being: direct relations and congruity effects Eur J Soc Psychol 30(2):177-198. https://doi. org/10.1002/(SICI)1099-0992(200003/04)30:23.0.CO;2-Z 
Schwartz SH (1992) Universals in the content and structure of values: theoretical advances and empirical tests in 20 countries. Adv Exp Soc Psychol. https:// doi.org/10.1016/S0065-2601(08)60281-6

Schwartz SH (2012) An overview of the schwartz theory of basic values. Online Readings Psychol Cult. https://doi.org/10.9707/2307-0919.1116

Schwartz SH, Bardi A (2001) Value hierarchies across cultures: taking a similarities perspective. J Cross Cult. Psychol https://doi.org/10.1177/0022022101032003002

Schwartz SH, Cieciuch J, Vecchione M, Davidov E, Fischer R, Beierlein C, Ramos A, Verkasalo M, Lonnqvist JE, Demirutku K, Dirilen-Gumus O, Konty M (2012) Refining the theory of basic individual values. J Pers Soc Psychol. https://doi.org/10.1037/a0029393

Schwartz SH, Melech G, Lehmann A, Burgess S, Harris M, Owens V (2001) Extending the cross-cultural validity of the theory of basic human values with a different method of measurement. J Cross Cult Psychol 32(5):519-542. https://doi.org/10.1177/0022022101032005001

Schwartz SH, Rubel T (2005) Sex differences in value priorities: cross-cultural and multimethod studies. J Pers Soc Psychol 89(6):1010-1028. https://doi.org/ 10.1037/0022-3514.89.6.1010

Schwartz SH, Sortheix F (2018) Values and subjective well-being. Handb WellBeing 1-25. https://jyx.jyu.fi/handle/123456789/56855

Schwartz S, Sagiv L (2000) Value priorities and subjective well-being: direct relations and congruity effects. Eur J Soc Psychol 30:177-198

Seddig D, Davidov E (2018) Values, attitudes toward interpersonal violence, and interpersonal violent behavior. Front Psychol. https://doi.org/10.3389/ fpsyg.2018.00604

Stanghellini G, Ballerini M (2007) Values in persons with schizophrenia. Schizophr Bull 33(1):131-141. https://doi.org/10.1093/schbul/sbl036

Sverdlik N, Oreg S (2015) Identification during imposed change: the roles of personal values, type of change, and anxiety. J Pers 83(3):307-319. https://doi. org/10.1111/jopy.12105

Talevi D, Rossi A (2020) Recovery and values in psychosis: is there a place for connection? J Psychopathol 26(2):119-120. https://doi.org/10.36148/22840249-385

Terracciano A, McCrae RR, Costa PT (2003) Factorial and construct validity of the Italian Positive and Negative Affect Schedule (PANAS). Eur J Psychol Assess 19(2):131-141. https://doi.org/10.1027//1015-5759.19.2.131

Treichler EBH, Li F, O’Hare M, Evans EA, Johnson JR, Spaulding WD (2019) Psychosocial and functional contributors to personal recovery in serious mental illness. J Ment Heal 28(4):427-435. https://doi.org/10.1080/ 09638237.2018 .1521932
Van Zelst C (2009) Stigmatization as an environmental risk in schizophrenia: a user perspective. Schizophr Bull 35(2):293-296. https://doi.org/10.1093/ schbul/sbn 184

Werner P, Aviv A, Barak Y (2008) Self-stigma, self-esteem and age in persons with schizophrenia. Int Psychogeriatr 20(1):174-187. https://doi.org/10.1017/ S1041610207005340

Zimmermann P, Firnkes S, Kowalski JT, Backus J, Siegel S, Willmund G, Maercker A (2014) Personal values in soldiers after military deployment: associations with mental health and resilience. Eur J Psychotraumatol. https://doi.org/ 10.3402/ejpt.v5.22939

\section{Competing interests}

The authors declare no competing interests.

\section{Additional information}

Correspondence and requests for materials should be addressed to V.S.

Reprints and permission information is available at http://www.nature.com/reprints

Publisher's note Springer Nature remains neutral with regard to jurisdictional claims in published maps and institutional affiliations.

(c) (i) Open Access This article is licensed under a Creative Commons Attribution 4.0 International License, which permits use, sharing, adaptation, distribution and reproduction in any medium or format, as long as you give appropriate credit to the original author(s) and the source, provide a link to the Creative Commons license, and indicate if changes were made. The images or other third party material in this article are included in the article's Creative Commons license, unless indicated otherwise in a credit line to the material. If material is not included in the article's Creative Commons license and your intended use is not permitted by statutory regulation or exceeds the permitted use, you will need to obtain permission directly from the copyright holder. To view a copy of this license, visit http://creativecommons.org/ licenses/by/4.0/.

(C) The Author(s) 2021 